\title{
A nálisis bibliométrico de los impresos peruanos relacionados a temas médicos publicados durante el Perú Virreinal (Siglos XVI-XIX)
}

\author{
ROY SARAVIA \\ Facultad de Medicina, Universidad Nacional Mayor de San Marcos.
}

\section{RESUMEN}

OBJETIVO: Cuantificar las características de los indicadores bibliométricos de los impresos relacionados a temas médicos publicados durante el Perú Virreinal (1585-1821). DISEÑ O: Estudio descriptivo, longitudinal y retrospectivo. MATERIAL Y MÉTODOS: En 550 impresos se evalúa los siguientes indicadores: título, año de publicación, autor, género documental, área temática, nacionalidad y profesión del autor, imprenta o impresor. RESU LTAD OS: Se halló 550 impresos entre los años 1585-1821, encontrándose una mayor frecuencia de publicación entre los años 1780-1799, con 280 (51\%) impresos. De 121 autores, Hipólito U nánue, con 72 (13,1\%) publicaciones, fue el más prolífico. De 32 áreas temáticas, la climatología, con 110 (20\%) impresos, fue la más recurrente. El género documental más empleado, con $192(34,9 \%)$ publicaciones, fue el libro. Los médicos, con $243(44,2 \%)$ impresos, tuvieron la mayor concentración de publicaciones. L a mayoría de impresos, $513(93,3 \%)$, fue publicada en castellano; 227 (41,3\%) impresos fueron publicados por autores peruanos. CON CL U SION ES: EI M ercurio Peruano se constituyó -cuantitativamente- en la publicación de mayor producción de literatura médica del Perú virreinal. Entre quienes allí publicaban, Hipólito U nánue fue el más prolífico de los autores, siendo la climatología la materia de mayor interés en la comunidad médica virreinal.

Palabras clave: Bibliometría; impresos sueltos; Perú. H istoria de la medicina del siglo XVI; Perú. Historia de la medicina del siglo XIX.

\section{BIBLIOMETRIC ANALYSIS OF MEDICAL RELATED PERUVIAN PRINTINGS PUBLISHED DURING PERUVIAN VICEROYALTY (XVI-XIX CENTURIES) SUMMARY}

OBJECTIVE: To quantify bibliometric indicators of medical printed matter published during Peruvian Viceroyalty (1585-1821). DESIGN : Retrospective, Iongitudinal, descriptive study. M ATERIAL AND METHODS: In 550 printings the following indicators were studied: title, year of publication, author, document gender, thematic area, author's nationality and profession, printing shop or printer. RESULTS: There were 550 printings between 1585-1821, 280 (51\%) published mainly between years 1780-1799. From 121 authors H ipolito U nanue was the most fruitful author with $72(13,1 \%)$ publications. From 32 thematic areas, climatology was the most recurrent with $110(20 \%)$ printings. The document gender mostly used was the book with $192(34,9 \%)$ publications. D octors had the largest concentration of publications with $243(44,2 \%)$ printings. M ost printings were published in Spanish, 513 (93,3\%), and 227 (41,3\%) by Peruvian authors. CON CL USIONS: The M ercurio Peruano was quantitatively the most published medical literature during Peruvian Viceroyalty. Hipolito Unanue was the most fruitful author, and climatology the matter of most interest in the Viceroyalty medical community.

Palabras clave: Bibliometrics; broadsides; Perú. H istory of medicine, 16th cent.; Perú. H istory of medicine, 19th cent.

Correspondencia:

Roy Saravia Mendoza

Calle Río Chira 471, La Viña

Lima 30, Perú

E-mail: roysm@latinmail.com 


\section{INTRODUCCIÓN}

A I llegar los españoles a A mérica, traían bajo sus celadas y adargas una medicina de corte medieval, galénico-arabizada, con todos los influjos de esta edad. Pero en la E uropa que ellos dejaban irrumpía la edad moderna, etapa con la cual convivió el virreinato peruano.

Bajo el nombre de renacimiento, no sólo entendemos el periodo en el cual reviven las ideas y conocimientos que habían sido logrados en la antigüedad clásica. Se trata, en verdad, de una superación de las ideas medioevales, con concepciones a veces en franca contradicción, en las que, además de recoger la herencia clásica, se reconoce la influencia de nuevos procesos técnicos y científicos. Fue la imprenta fruto del renacimiento, la que rompió el lazo de subordinación del alumno al maestro, al permitir a cada uno el diálogo con el libro multiplicado en millares de copias ( $\left.{ }^{1}\right)$.

El primer libro impreso en el $\mathrm{N}$ uevo $\mathrm{M}$ undo, publicado en M éxico (1537), fue La escala espiritual para llegar al cielo, escrito por Fray Juan de la Madalena, que tradujo del latín la obra de San Juan Clímaco $\left({ }^{2}\right)$. El primer libro impreso en el Perú fue La Doctrina cristiana y Catecismo para La Instrucción de los Indios (3), publicado en 1584, a cargo del primer impresor de Lima, A ntonio Ricardo. Como se ve, bastan estos dos títulos para dar una idea de cuál era la sustancia de la producción impresa en A mérica en el siglo XVI, plagada de tratados ascéticos, estudios morales, instrumentos doctrinales, regulaciones eclesiásticas y hagiografías, situación que iría cambiando lentamente a lo largo de los dos siglos siguientes, según el camino intelectual trazado por Europa. En forma reposada penetrarían las nuevas corrientes de pensamiento en el Perú, anidándose en bibliotecas conventuales y particulares, desde donde sólo se difundirían a las clases sociales privilegiadas.

Es evidente que el análisis de las publicaciones científico-médicas aparecidas en el Perú virreinal es delicado y parcial, pues sólo representa una parte de la actividad científica gene- ral, pero es un buen reflejo de la estructura mental de los distintos grupos socio-profesionales que integraban la comunidad médica de este periodo.

El análisis bibliométrico, como método documental, nos permite esencialmente acercarnos al estudio del volumen, crecimiento y distribución de los documentos médicos impresos, así como la indagación de la estructura social y dinámica de los grupos que producen dichos documentos; es decir, la bibliometría realiza un análisis estadístico y sociométrico de la bibliografía científica ( $\left.{ }^{4-6}\right)$.

El presente estudio tiene como objetivo determinar las características de los indicadores bibliométricos de los impresos peruanos sobre temas médicos, que fueron publicados durante el virreinato.

\section{MATERIAL Y MÉTODOS}

El tipo de estudio del presente trabajo es retrospectivo, longitudinal, descriptivo y de observación, cuyo universo es el total de impresos peruanos relacionados a temas médicos, publicados durante el Perú Virreinal (1585-1821).

Las fuentes primarias fueron: 1.-Colecciones Peruanas del M useo de la Facultad de M edicina de San Fernando de la U NM SM ; 2.-M ercurio Peruano Tomos I-XI, 1791-1795. Edición Facsimilar, Biblioteca $\mathrm{N}$ acional del Perú. Lima, 1964-1966. L as fuentes secundarias fueron: 1.La Imprenta de Lima. Medina José Toribio. Tomos I-III. Santiago de Chile, 1904; 2.-Biblioteca Peruana- Impresos Peruanos. Vargas U garte Rubén. Tomos VII-XII. Lima, 1957; 3.-A puntes para la Bibliografía M édica Peruana. Val dizán Hermilio. Lima, 1928.

Fueron elegibles para el estudio todos los impresos peruanos relacionados a temas médicos, implícita o tácitamente, publicados durante el periodo 1585-1821. Fueron excluidos los impresos peruanos atribuidos al periodo en estudio, pero sin fecha exacta de publicación. 
Con respecto a la técnica e instrumento de recolección de datos, se hizo uso del análisis bibliométrico y de una ficha de recolección de datos para cada impreso, en la cual se consignó los siguientes indicadores: título, año de publicación, autor, género documental del impreso, área temática del impreso, nacionalidad del autor, profesión del autor y el impresor o la imprenta.

Para el procesamiento y análisis de los datos, se empleó una computadora personal IBM de $266 \mathrm{M} \mathrm{hz}$, con la base de datos D-Base IV, y el paquete estadístico E pi Info 2000 para la estadística descriptiva.

\section{RESULTADOS}

En total se estudió 550 impresos, abarcando un periodo de 237 años (1585-1821) ( $\left.{ }^{7}\right)$.

L a mayor frecuencia de impresos se encontró entre los años 1780-1799, con un total de $280(51 \%)$ publicaciones (Tabla 1$)$.

Tabla 1. Número de impresos peruanos relacionados a temas médicos publicados en el Perú Virreinal, según año de publicación.

\begin{tabular}{|c|c|c|}
\hline \multirow[t]{2}{*}{ Año de publicación } & \multicolumn{2}{|c|}{ I mpresos peruanos } \\
\hline & $n$ & $\%$ \\
\hline $1585-1599$ & 1 & 0,2 \\
\hline $1600-1619$ & 5 & 0,9 \\
\hline $1620-1639$ & 5 & 0,9 \\
\hline $1640-1659$ & 5 & 0,9 \\
\hline $1660-1679$ & 2 & 0,4 \\
\hline $1680-1699$ & 16 & 2,9 \\
\hline $1700-1719$ & 3 & 0,5 \\
\hline $1720-1739$ & 22 & 4,0 \\
\hline $1740-1759$ & 31 & 5,6 \\
\hline $1760-1779$ & 38 & 6,9 \\
\hline $1780-1799$ & 280 & 51,0 \\
\hline $1800-1821$ & 142 & 25,8 \\
\hline Total & 550 & 100,0 \\
\hline
\end{tabular}

Se encontró un total de 121 autores, teniendo H ipólito U nánue $72(13,1 \%)$ publicaciones, el sacerdote Francisco Romero 53 (9,6\%) publicaciones, Cosme Bueno 51 (9,3\%) publicaciones (Tabla 2).

Tabla 2. Número de publicaciones según autores de los impresos peruanos en el Perú Virreinal.

\begin{tabular}{lrr}
\hline \multicolumn{1}{c}{ Autor } & $\begin{array}{c}\text { Número de publicaciones } \\
\mathrm{n}\end{array}$ & $\%$ \\
\hline Unanue, Hipólito & 72 & 13,1 \\
Romero, Francisco & 53 & 9,6 \\
Bueno, Cosme & 51 & 9,3 \\
Peral ta Barnuevo, Pedro & 21 & 3,8 \\
Moreno, Gabriel & 15 & 2,7 \\
Paredes, Gregorio & 14 & 2,5 \\
Rossi y Rubí, J osé & 13 & 2,4 \\
Val dés, J oséManuel & 11 & 2,0 \\
Otros & 176 & 32,0 \\
Indeterminado & 124 & 22,5 \\
Total & 550 & 100,0 \\
\hline
\end{tabular}

En lo referente al área temática de los impresos, se halló 32 temas diferentes, siendo el más recurrente la climatología, con $110(20 \%)$ publicaciones, seguido por la terapéutica, con 37 $(6,7 \%)$ publicaciones, entre otras (Tabla 3$)$.

Tabla 3. Á rea temática de los impresos peruanos.

\begin{tabular}{|c|c|c|}
\hline \multirow[t]{2}{*}{ Á rea temática } & \multicolumn{2}{|c|}{ I mpresos peruanos } \\
\hline & $\mathrm{n}$ & $\%$ \\
\hline Climatología & 110 & 20,0 \\
\hline Terapéutica & 37 & 6,7 \\
\hline Astronomía & 37 & 6,7 \\
\hline Meteorología & 25 & 4,5 \\
\hline Infectología & 14 & 2,5 \\
\hline Otros & 327 & 59,6 \\
\hline Total & 550 & 100,0 \\
\hline
\end{tabular}

Entre los tipos de género documental hallados, $192(34,9 \%)$ fueron libros, $182(33,1 \%)$ artículos de revistas, $79(14,4 \%)$ artículos de periódico, $46(8,4 \%)$ folletos, $24(4,4 \%)$ pape- 
les y $19(3,5 \%)$ tesis. En $8(1,5 \%)$ no se pudo determinar el género documental.

Entre las profesiones de los autores, se encontró médicos con $243(44,2 \%)$ publicaciones, sacerdotes con $76(13,8 \%)$ publicaciones, abogados con $26(4,7 \%)$, entre otros (Tabla 4$)$.

Tabla 4. Profesión de los autores de los impresos peruanos.

\begin{tabular}{lrr}
\hline \multicolumn{1}{c}{ Profesión } & \multicolumn{2}{c}{ Impresos peruanos } \\
& $\mathrm{n}$ & $\%$ \\
\hline Abogado & 26 & 4,7 \\
Dentista & 1 & 0,2 \\
Escritor & 3 & 0,5 \\
Farmacéutico & 1 & 0,2 \\
Funcionario & 13 & 2,4 \\
Médico & 243 & 44,2 \\
Militar & 9 & 1,6 \\
Naturalista & 1 & 0,2 \\
Sacerdote & 76 & 13,8 \\
Indeterminado & 177 & 32,2 \\
Total & 550 & 100,0 \\
\hline
\end{tabular}

De los impresos, $513(93,3 \%)$ fueron publicados en castellano, $36(6,5 \%)$ en latín, encontrándose además una publicación bilingüe castellano francés; $227(41,3 \%)$ impresos fueron publicados por autores peruanos, $66(12,3 \%)$ por autores españoles, además de otros autores de diversas nacionalidades en menor proporción (Tabla 5).

Tabla 5. Nacionalidad de los autores de los impresos peruanos.

\begin{tabular}{lrr}
\hline Nacionalidad & \multicolumn{2}{c}{ Impresos peruanos } \\
& $\mathrm{n}$ & $\%$ \\
\hline Peruana & 227 & 41,3 \\
Española & 66 & 12,0 \\
Checoesl ovaca & 9 & 1,6 \\
Francesa & 2 & 0,4 \\
Italiana & 1 & 0,2 \\
Indeterminada & 245 & 44,5 \\
Total & 550 & 100,0 \\
\hline
\end{tabular}

De un total de 26 impresores o imprentas encontradas, Ia Imprenta de los $\mathrm{N}$ iños Expósitos realizó $85(15,5 \%)$ de las publicaciones, la Imprenta Real $25(4,5 \%)$, el impresor Bernardino Ruiz $10(1,8 \%)$, seguido por otros en menor magnitud .

L a población de Lima varió lentamente, desde 1600 con 14,262 habitantes hasta 1818 con 54,098 habitantes $\left({ }^{8,9}\right)$; su asociación con la evolución del número de impresos durante el mismo periodo se presenta en el Figura 1.

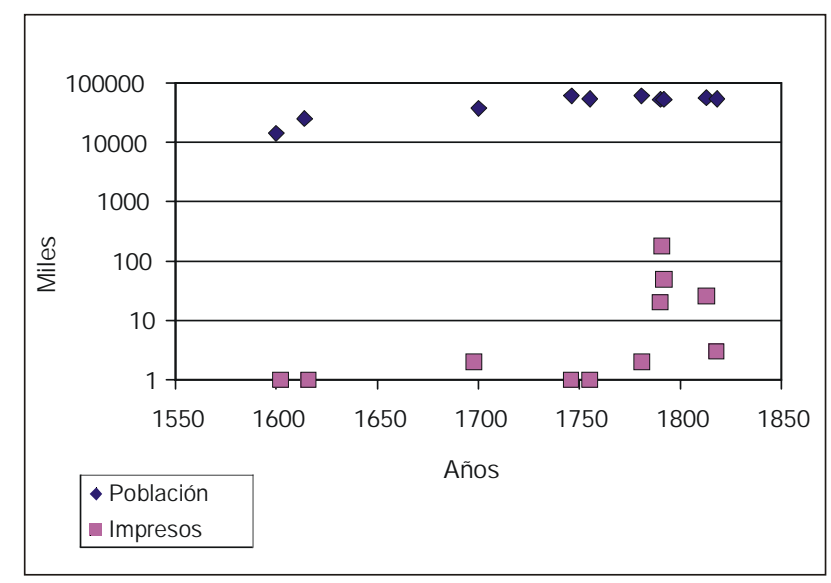

Figura 1. Impresos peruanos relacionados a temas médicos y población de Lima (1600-1818).

\section{DISCUSIÓN}

Se identificó un total de 550 impresos durante el periodo en estudio. La mayor concentración de impresos por año se encuentra entre 1780 1799 , con $280(51 \%)$ del total de publicaciones, con una media de 28 publicaciones por año. Esto es debido al impulso exponencial ejercido por el M ercurio Peruano, al cual pertenecen 182 de estas publicaciones. Es un hecho muy conocido la repercusión que tuvo esta revista en la vida intelectual de finales del siglo XVIII ${ }^{\left({ }^{10}\right)}$. Sin embargo, hasta ahora no se disponía de una prueba cuantitativa que expresara la magnitud de tal influencia. El M ercurio Peruano -surgido del entusias- 
mo intelectual de la Sociedad Académica de A mantes del País en vínculo con el Virrey Gil de Taboada y Lemos-, durante sus cuatro años de existencia (1791-1794) fue el paradigma de la ilustración en el Perú virreinal. Su contenido podría equipararse al de la Enciclopedia francesa, porque en sus páginas hicieron eco los más diversos géneros de la ciencia $E$ uropea y N orteamericana de la época. Además, el M ercurio nunca soslayó la cultura nacional, dedicando holgadas páginas a revisar la variopinta realidad peruana, a través de artículos científicos, históricos, políticos, literarios, etc. Por ello, no es extraño que el Mercurio publicara el discurso de inauguración del A nfiteatro A natómico, dado por $\mathrm{H}$ ipólito U nanue con el título "D ecadencia y restauración del Perú" $\left.{ }^{11}\right)$, celebrado el 21 de noviembre de 1782, en la Real Universidad de San M arcos. En él se analiza la relación entre el deterioro económico del país y la deficiencia de la atención médica; alocución sobre la necesidad de mejorar la enseñanza médica en el Perú, como una exigencia inel uctable para salir del atraso económico y social.

El primer libro sobre tema médico sensu estricto, identificado por nosotros, es: Dos tratados uno de las cualidades y affetos de la alaxa y otro de una especie de garrotillo o esquinancia mortal (12), escrito por Francisco de Figueroa en 1616, no concordando con Valdizán, quien en La Facultad de Medicina de Lima (13) menciona como la primera publicación médica en el Perú la Suma y recopilación de cirugía, por Alfonso López, impresa en 1578, confusión surgida porque este libro fue impreso por Antonio Ricardo en M éxico, antes de llegar al Perú, donde se convertiría luego en el primer impresor.

De un total de 121 autores, Hipólito U nanue fue el más prolífico, con $72(13,1 \%)$ del total de publicaciones. Valdizán, en la Facultad de Medicina de Lima, encuentra desde la primera publicación de U nanue en el M ercurio Peruano, en 1791 y hasta 1821, sólo 69 publicaciones. Unanue fue una figura preeminente de la vida científica y cultural peruana de finales del siglo XVIII y las primeras décadas del siglo XIX. Fueron producto de sus afanes la fundación del
Real A nfiteatro A natómico de San A ndrés y la creación del Colegio de Medicina de San Fernando. Ejerció el cargo de Cosmógrafo $M$ ayor del Reino y regentó la cátedra de anatomía de la Real y Pontificia Universidad de San M arcos. Fue U nanue piedra angular de la ilustración en el Perú virreinal. Su vasta producción intelectual -reflejada aquí cuantitativamente-, sin temor a conjeturas, expresa el espíritu de modernidad que impelía como secretario de la Sociedad Académica de A mantes del País.

EI área temática de mayor recurrencia fue la climatología, con 110 (20\%) impresos dedicados a este tema. Hay que mencionar que la astrología, en un principio, luego la meteorología, la climatología y finalmente la astronomía estuvieron estrechamente relacionadas a los posibles efectos que pudieran tener sobre el proceso de salud-enfermedad, persistiendo este concepto hasta llegar a la ópera magna de U nanue Observaciones sobre el clima de Lima y su influencia sobre los seres organizados, en especial el hombre $\left({ }^{14}\right)$, publicada en 1806 . Esta obra es considerada como la más importante escrita en las postrimerías del virreinato, donde, con sutil y elegante prosa, U nanue describe en las primeras cuatro secciones la historia del clima, las influencias del clima, las influencias del clima sobre las enfermedades y los medios de curar las enfermedades del clima. El contenido argumental de las Observaciones sobre el clima de Lima, sólidamente sostenido en autores clásicos, como H ipócrates, Sydenham, Boerhaave, Stoll, Haen y Jakson, le hacen decir a Lastres que un mejor titulo para esta obra sería "Manual M édico Filosófico de Clima, Patología y Terapéutica de Lima y sus alrededores" (15). Recordando que Unanue fue Cosmógrafo $\mathrm{Ma}$ ayor del Reino, debemos aquí subrayar que, con frecuencia, este cargo de cosmógrafo -encargado de publicar los almanaques Ilamados primero Conocimiento de los Tiempos, luego Guía de Forasteros y por último Guía política, eclesiástica y militar y que aparecieron en Lima desde 1721 hasta 1874- era ejercido por un médico. Bástenos señalar a Cosme Bueno, en cuya biblioteca, junto a clásicos de la medicina, se 
encuentran tratados de matemática, astronomía y climatología de la más diversa índole ${ }^{\left({ }^{16}\right)}$. A sí mismo, habitualmente, el cosmógrafo también ejercía la cátedra de matemáticas en la U niversidad de San M arcos. Recordemos que en las enciclopedias de matemáticas de la época, la astronomía, astrología, meteorología y la cosmografía se encontraban en el apartado de matemáticas impuras o aplicadas. M édicos como Cosme Bueno y José Gregorio Paredes fueron, a su vez, catedráticos de matemáticas y cosmógrafos.

El género documental de mayor presentación fue el libro, con un total de 192 (34,9\%) impresos, seguido por los artículos de revista, con $182(33,1 \%)$, que pertenecieron en su totalidad al Mercurio Peruano. Los artículos de periódico, $79(14,4 \%)$ pertenecieron al Diario de Lima, EI Verdadero Peruano y EI I nvestigador, siendo el más importante el primero, fundado por Jaime Bauzate y M eza, el $1^{\circ}$ de octubre de 1790 $\left.{ }^{17}\right)$. Los folletos fueron $46(8,4 \%)$, la mayor parte opúsculos y breviarios. Los papeles impresos, $24(4,4 \%)$, correspondientes en su mayoría a anuncios, besamanos, etc. $L$ as tesis fueron $19(3,5 \%)$, todas ellas para optar el grado de bachiller en medicina.

La profesión más frecuente de los cultivadores de la ciencia médica fue obviamente la de los médicos, con $243(44,2 \%)$ impresos, dato este concordante con lo encontrado por López Piñero ${ }^{(18)}$, para quien, entre todos los cultivadores de la ciencia en general, los médicos representan la mayoría. Los sacerdotes se encuentran en el segundo lugar, debido a que en los primeros años del virreinato, ante la escasez de médicos, era el clero el que se ocupaba de estos menesteres, regentando primero boticas parroquiales y luego impulsando la creación de hospitales, así como, su administración a través de órdenes religiosas. Precisemos, además que, durante el virreinato, ante la escasez de bibliotecas públicas, eran los colegios y conventos las que tenían el mayor fondo bibliográfico. Entre las que podemos citar está la biblioteca del Colegio de San Pablo, tutelado por los jesuitas que, para la segunda mitad del siglo XVII, contaba con casi 40,000 volúmenes, considerada para entonces como la mejor biblioteca de A mérica $\left({ }^{19}\right)$.

El idioma de mayor uso, como era de esperarse, fue el castellano, en $513(93,3 \%)$ de las publicaciones. El latín fue usado en $36(6,5 \%)$, siendo éste de uso obligatorio en todas las tesis. Y, por último, encontramos una publicación bilingüe en castellano - francés, que fue la impresión de la tesis de José M anuel Dávalos para graduarse de doctor en la U niversidad de M ontpellier, en $1787\left({ }^{20}\right)$.

A unque al inicio del virreinato la totalidad de publicaciones fue hecha por autores españoles, las cosas se equipararían al final, siendo los nacidos en nuestra tierra los que más publicaron, con 227 (41,3\%) impresos; esto se debió a que los primeros miembros de la comunidad médica virreinal, todos provenían de universidades de la península. Sólo con la creación de la U niversidad de San M arcos, se empezaría a formar médicos peruanos. D ebemos apuntar que el inicio - oficial aunque no real- de la enseñanza médica en el Perú esta fechado en 1571, año de la aparición de la primeras "Constituciones de la U niversidad de los Reyes", en cuyo artículo 15, titulado "Bachiller en medicina", señala que se debía seguir tres años de estudio y defender seis conclusiones sacadas de la Articella de Galeno y el Canon de Avicena, para poder graduarse $\left({ }^{21}\right)$. Pero, el establecimiento de la enseñanza científica de la medicina sólo se daría a partir del 13 de agosto de 1808, en que $\mathrm{H}$ ipólito U nanue suscribe el "Quadro sinóptico de las ciencias que se enseñan en el Colegio de M edicina de San Fernando de Lima..." ( $\left.{ }^{7}\right)$, verdadero legado pedagógico de la voluntad progresista de U nanue.

La Imprenta de los $\mathrm{N}$ iños Expósitos fue la mayor productora de publicaciones del Perú virreinal. En 1758 dio a la estampa su primera publicación, manteniéndose con ciertos altibajos hasta 1824, año en que desaparece ( ${ }^{22}$ ).

En cuanto a la evolución del número de publicaciones respecto a la población de Lima, no existe una correlación lineal entre ambas. A la 
evolución demográfica de la población limeña, no siguió como sería de esperarse un aumento de publicaciones en igual proporción. Esto es un indicio de la escasa o nula política cultural virreinal, atribuible también al exiguo nivel de instrucción de la mayoría de nuestra población y, por último, al progresivo deterioro económico de las colonias españolas en A mérica. Para 1790, Lima contaba con 52,627 habitantes; la élite intelectual sólo incluía 21 médicos, 91 abogados, 366 estudiantes, 56 cirujanos, 13 notarios y 58 escribanos $\left({ }^{23}\right)$. E sto significa una parva proporción de la población total, por lo que fácil es comprender que el consumo de publicaciones no fuera una actividad socialmente muy difundida. La singular aparición del Mercurio, que elevó en forma exponencial el número de publicaciones, durante un corto periodo, surgió, según Fisher $\left.{ }^{23}\right)$, de "el tardío énfasis borbónico en la adquisición de un conocimiento útil, una investigación racional y el crecimiento económico", impulso que luego de desaparecida la revista, en el remanente del virreinato, no volvería a alcanzar enjundiosidad igual.

En conclusión, el M ercurio Peruano se constituyó -cuantitativamente- en la publicación de mayor producción de literatura médica del Perú virreinal. Entre los que allí publicaban, $\mathrm{H}$ ipólito Unanue fue el más prolífico de los autores, siendo la climatología la materia de mayor interés en la comunidad médica virreinal.

Esta información así obtenida, al ser agregada a los condicionamientos sociales presentes en la época, nos permitirá finalmente tener una visión totalizadora de los productores y productos de la actividad científico-médica virreinal.

\section{BIBLIOGRAFÍA}

1. Mieli A. La eclosión del renacimiento. 2ra Edición. M adrid:Espasa-C alpe S. A, 1967.

2. García I zcalbalzeta J . Bibliografía mexicana del siglo XVI. 1a Edición. M éxico: Fondo de Cultura E conómica; 1954.

3. Baudot $G$. La vida cotidiana en la A mérica española en tiempos de Felipe II. Siglo XVI. $1^{\text {a }}$ edición en español. M éxico: Fondo de Cultura E conómica, 1983.

4. López Yepes P. Introducción a la bibliometría. Valencia:Promolibro, 1996.
5. González de Dios, M oya M. Indicadores bibliométricos: Características y limitaciones en el análisis de la actividad científica. An Esp Pediatr 1997; 49: 235-44.

6. González de Dios J, M oya M. Estudio bibliométrico de "A nales Españoles de Pediatría" (Década 1984-1993) I: A nálisis de los artículos publicados. An Esp Pediatr 1997; 42: 2-10.

7. Saravia M endoza R. A nálisis bibliométrico de los impresos peruanos relacionados a temas médicos publicados durante el Perú virreinal (siglos XVI-XIX) [Tesis de bachiIler]. Lima:U niversidad Nacional M ayor de San M arcos; 1999.

8. Reflexiones históricas y políticas sobre el estado de la población de esta capital, que se acompaña por suplemento. M ercurio Peruano 1791; 10: Febrero. E dición Facsimilar, Biblioteca N acional del Perú, Lima, 1964.

9. Gootemberg P. Población y Etnicidad en el Perú Republicano. Lima:Instituto de Estudios Peruanos. Documento de Trabajo N 71; 1995.

10. Mariátegui J. EI Mercurio Peruano y la Medicina. L ima:U niversidad Peruana Cayetano Heredia, Fondo Editorial; 1994.

11. Decadencia y restauración del Perú. Oración inaugural que para la extrena y abertura del A nfiteatro A natómico dixo en la Real U niversidad de San M arcos el día 21 de noviembre de 1792, el D octor D on J oseph H ipólito U nanue, C atedrático de A natomía y Secretario de la Sociedad [A cadémica de A mantes del País]. M ercurio Peruano 1792;218, 219,220,221: M ayo, Junio, Julio, A gosto. E dición Facsimilar, Biblioteca Nacional del Perú, Lima, 1964.

12. Valdizán H. A puntes para la bibliografía médica peruana. L ima:I mprenta A mericana; 1928.

13. Valdizán H. La Facultad de M edicina de Lima. 2a edición, Tomo II. Lima; 1928.

14. Unanue H. Observaciones sobre el clima de Lima y sus influencias en los seres organizados, en especial el hombre. L ima:Imprenta Real de los Huérfanos; 1721.

15. Paz-Soldán CE. Himnos a Hipólito U nanue. L ima:Editorial San M arcos; 1955.

16. Saravia M endoza R. La biblioteca del Dr. Cosme Bueno: A nálisis bibliométrico e inventario. Inédito.

17. M oro Álvarez J. H istoria de la prensa hispanoamericana. M adrid:E ditorial M APFRE; 1992.

18. López Piñero J M, Glick TF. Bibliometría e Inventario. Valencia:H ispaniae Scientia; 1979.

19. Martin L. La biblioteca del Colegio de San Pablo (15681767), antecedente de la Biblioteca $N$ acional. F énix: Revista de la Biblioteca N acional 1971; 21: 25-37.

20. Lastres J B. Historia de la M edicina Peruana. Lima: Imprenta Santa M aría; 1951.

21. Eguiguren LA. La Universidad en el Siglo XVI, Tomo I. Lima:Imprenta Santa M aría; 1951.

22. M edina J T. La imprenta en L ima. Santiago de Chile: Impreso y grabado en casa del autor; 1904.

23. Fisher J. El Perú Borbónico 1750-1824. Lima:Instituto de Estudios Peruanos; 2000. 